Pbilosophia Scientie

\section{Philosophia Scientiæ}

Travaux d'histoire et de philosophie des sciences

9-2| 2005

Aperçus philosophiques en logique et en mathématiques

\title{
De Kant à Gentzen, en passant par Brouwer, Hilbert et Frege
}

\section{Pierre Cassou-Noguès}

\section{(2) OpenEdition \\ Journals}

Édition électronique

URL : http://journals.openedition.org/philosophiascientiae/538

DOI : 10.4000/philosophiascientiae.538

ISSN : 1775-4283

Éditeur

Éditions Kimé

Édition imprimée

Date de publication : 1 novembre 2005

Pagination : 205-223

ISBN : 2-84174-379-9

ISSN : $1281-2463$

Référence électronique

Pierre Cassou-Noguès, " De Kant à Gentzen, en passant par Brouwer, Hilbert et Frege », Philosophia Scientiæ [En ligne], 9-2 | 2005, mis en ligne le 15 juin 2011, consulté le 15 janvier 2021. URL : http:// journals.openedition.org/philosophiascientiae/538 ; DOI : https://doi.org/10.4000/ philosophiascientiae.538 


\title{
Le temps, l'espace et la démonstration. De Kant à Gentzen, en passant par Brouwer, Hilbert et Frege
}

\author{
Pierre Cassou-Noguès \\ CNRS, UMR 8519 (Lille 3) Savoirs et Textes
}

Résumé : Le but de cet article est d'étudier la référence à l'espace et au temps dans le problème du fondement des mathématiques, au cours de la période 1880-1935. Après avoir évoqué la problématique kantienne, qui reste présente dans la controverse entre Brouwer et Hilbert, nous discutons de la référence au temps dans l'intuitionisme et dans le programme formaliste pour montrer comment, dans les deux cas mais de façon différente, la référence au temps introduit des restrictions sur ce qui peut être considéré comme une démonstration mathématique. Nous évoquons ensuite trois tentatives, Frege, le Hilbert d'avant le programme formaliste et Gentzen, pour éliminer la référence au temps et ne fonder les mathématiques que sur l'intuition de l'espace.

Abstract: The aim of this paper is to analyse the reference to space and time in the foundation of mathematics. First, we describe the reference to time in Brouwer's intuitionism and in Hilbert's formalism in order to show that the reference to time leads to different restrictions on what can be considered a mathematical proof. We then study three attempts, by Frege, by Hilbert around 1900, by Gentzen, to eliminate the reference to time and ground mathematics on the intuition of space. 
J'examinerai le rôle de l'espace et du temps dans le fondement des mathématiques. Si l'on considère les preuves, les démonstrations, comme les actes d'un sujet, on dira qu'elles se déploient dans le temps, si elles sont pensées, et dans l'espace, si elles sont écrites. L'insertion dans l'espace et dans le temps devient un fondement pour les preuves mathématiques mais, du même coup, leur impose des restrictions. C'est cette double fonction de la référence à l'espace et au temps, fondement et restriction, que je tenterai de retracer, en évoquant quelques étapes de l'histoire de la logique depuis Frege.

Dans un article récent, G. Longo discute des travaux de J.-Y. Girard, qui, avec la logique linéaire, puis la logique "ludique", réintroduit des considérations géométriques dans l'analyse des preuves [Longo 2003]. Ces considérations géométriques, selon G. Longo, manquaient dans la controverse sur le problème des fondements et cela explique l'impasse à laquelle elle a abouti. Mon but serait de montrer que, en effet, l'intuitionisme et le formalisme, rapportent, quoique de façon différente, le raisonnement mathématique au temps et en tirent des restrictions, là encore différentes, pour les possibilités mathématiques. Néanmoins, je voudrais insister sur une série de tentatives, depuis Frege et son idéographie, pour dégager le raisonnement mathématique de l'inscription dans le temps et des restrictions qu'elle impose.

Je commencerai par rappeler la position de Kant, qui, on le sait, appuie les jugements mathématiques sur l'intuition de l'espace et du temps et qui restera un point de repère dans les discussions sur le problème des fondements. Je suivrai ensuite les solutions de Brouwer et de Hilbert, pour mettre en évidence la place, tout à fait différente, qu'ils donnent à la référence au temps. Enfin, j'évoquerai trois tentatives pour éliminer la référence au temps : celle de Hilbert, antérieurement à son programme de fondement, celle de Frege, qui se trouve faire face à un problème analogue au moment de son idéographie, et celle de Gentzen, avec les déductions naturelles.

\section{2}

On le sait, Kant justifie les jugements mathématiques par des constructions dans l'espace et dans le temps. Le point de départ est la distinction entre jugements analytiques (où le prédicat est contenu dans le concept du sujet : un homme grand est grand) et jugements synthétiques où le 
prédicat n'est pas contenu dans le concept du sujet. Les jugements mathématiques, " $5+7=12$ ", "la somme des angles d'un triangle est égale à un angle plat", sont synthétiques. Un triangle est une figure fermée à trois côtés. On peut, soutient Kant, analyser ce concept, on n'en déduira pas la mesure de la somme des angles. Une analyse des concepts ne suffit pas à établir les jugements mathématiques. Ceux-ci sont synthétiques et, pourtant, a priori. Le problème, à l'origine de la Critique de la raison pure, est de rendre compte de la possibilité de tels jugements.

Puisque le prédicat n'est pas contenu dans le concept du sujet, il faut que la prédication repose sur une base extérieure. Ce sera une construction dans l'intuition. Pour établir que la somme des angles d'un triangle est égale à deux droits, on trace un triangle et une droite passant par l'un des sommets et parallèle au côté opposé. On montre sur ce dessin que la somme des angles du triangle coïncide à un angle plat. Ainsi, la démonstration d'une proposition géométrique repose sur une construction dans l'espace, un dessin.

Les propositions arithmétiques exigent une construction dans le temps. Pour calculer la somme $5+7$, on commence par aligner cinq objets, disons cinq pommes, puis à côté sept autres pommes et on compte douze pommes au total. Mais, dans ce processus, la nature particulière des choses que l'on compte, les pommes, n'intervient pas. Seule importe la succession des objets dans l'acte de compter, c'est-à-dire la temporalité de l'acte. La construction, qui établit " $5+7=12$ ", ne repose que sur notre temporalité intérieure, sur cette forme temporelle que nous prêtons à notre vie mentale. En ce sens, les propositions arithmétiques reposent sur des constructions dans le temps.

Ainsi, Kant fonde les jugements mathématiques sur l'intuition de l'espace et du temps. La validité des propositions mathématiques ne tient qu'au caractère a priori de l'espace et du temps, en tant que formes des intuitions externes et internes. La position kantienne sera la référence privilégiée de Brouwer et de Hilbert. La controverse sur le problème des fondements entre l'intuitionnisme, autour de Brouwer, et le formalisme, autour de Hilbert, est liée à la découverte de paradoxes au tournant du XXe siècle. Ceux-ci engagent les mathématiciens à une critique des raisonnements classiques. Cette critique, de plus en plus radicale, conduira à l'intuitionnisme de Brouwer. Hilbert, avec son école de Göttingen, répondra à l'intuitionnisme en s'efforçant de donner un fondement aux mathématiques classiques. 
Or la référence au temps est un trait essentiel de la critique intuitionniste. Les propositions mathématiques reposent sur des raisonnements qui s'effectuent dans la conscience et, par conséquent, dans le temps. Cette temporalité semble imposer des restrictions sur nos raisonnements. Prenons l'exemple du tiers exclu. En arithmétique, le tiers exclu permet d'affirmer, P étant une propriété quelconque, que ou bien tous les entiers vérifient la propriété $\mathrm{P}$ ou bien il existe un entier qui ne vérifie pas la propriété P. Et, dans un raisonnement par l'absurde, lorsque l'on prouve que l'hypothèse que tous les entiers possèdent $\mathrm{P}$ conduit à une contradiction, on déduit l'existence d'un entier ne possédant pas P. Pourtant, au moment où l'on affirme l'existence d'un tel entier, on ne sait pas le calculer. Si l'on voulait vérifier l'existence de cet entier et, par là, établir la validité de l'inférence, il faudrait tester successivement chaque entier, jusqu'à en trouver un qui ne possède pas la propriété P. Mais il est possible que, aussi loin que l'on aille, tous les entiers que l'on teste possèdent la propriété P. Puisque l'on ne peut pas parcourir en totalité la suite des entiers, on ne peut pas vérifier qu'il existe un entier ne possédant pas la propriété P. Notre raisonnement par l'absurde ne semble pas légitime. Au moins, on ne peut pas le vérifier par une construction dans le temps. En réalité, pour justifier notre raisonnement par l'absurde, il faudrait postuler que la suite des entiers existe en soi et possède des propriétés déterminées, $\mathrm{P}$ ou non $\mathrm{P}$, indépendamment de nos calculs. Il faudrait postuler que la suite, infinie, des entiers existe à la façon d'un système fini d'objets dont, en effet, on peut affirmer ou bien que chacun possède la propriété $\mathrm{P}$ ou bien qu'il en existe un ne possédant pas la propriété P. Ainsi, les mathématiques classiques reposent sur l'hypothèse de l'infini actuel. Mais, après les paradoxes, il faut renoncer à cette hypothèse. Les énoncés mathématiques doivent pouvoir être justifiés par une construction dans la conscience et, par conséquent, une construction dans le temps. Les mathématiques classiques ne respectent pas ce principe. L'intuitionnisme les rejette.

On trouverait une analyse analogue et une référence au temps, moins explicite, dans certains textes de Borel et, par exemple, dans la critique que fait Borel de la démonstration par Zermelo du bon ordre à partir de l'axiome du choix. Borel caricature le raisonnement de Zermelo : "Pour bien ordonner un ensemble $M$, il suffit d'y choisir arbitrairement un élément auquel on attribuera le rang 1, puis un autre auquel on attribuera le rang 2, et ainsi de suite transfiniment" Et, ajoute Borel, "aucun mathématicien ne regardera comme valable ce dernier raisonnement" [Borel 1905, 295]. Cette évidence repose sur le postulat qu'un raisonnement se déroule dans le temps et que, dans le temps, ne peuvent s'accomplir que 
des opérations indéfinies, mais non transfinies.

L'intuitionnisme fonde les mathématiques sur l'intuition du temps. Pour Brouwer, l'existence des géométries non euclidiennes oblige à refuser la thèse kantienne d'une fondation de la géométrie dans l'intuition de l'espace. On peut prouver aussi bien des propositions qui portent sur des objets appartenant à un espace euclidien qu'à un espace non euclidien. La géométrie ne peut pas être fondée sur des constructions dans un espace déterminé, qu'il soit euclidien ou non euclidien. En revanche, Brouwer reprend la thèse d'une fondation de l'arithmétique et, en fait, des mathématiques sur l'intuition du temps. L'intuitionnisme "a pu se relever par l'abandon de l'apriorité kantienne de l'espace et le maintien d'autant plus ferme de l'apriorité du temps" [Brouwer 1912, 43]. Ici, le raisonnement est situé dans le temps, lequel représente un a priori assurant la légitimité des constructions mais imposant des restrictions sur les possibilités mathématiques. Restrictions, qui conduisent Brouwer à rejeter les mathématiques classiques.

Hilbert répond à l'intuitionnisme en s'efforçant de donner un fondement définitif aux mathématiques classiques. Brouwer, après Poincaré, montrait que les mathématiques classiques, telles qu'elles sont habituellement présentées, reposent sur l'hypothèse de l'infini actuel, l'hypothèse que les systèmes infinis existent à la façon des systèmes finis. Hilbert s'accorde avec Brouwer pour refuser cette hypothèse : "l'infini n'est nulle part réalisé ; il n'existe pas dans la nature et on ne peut pas l'admettre en tant que fondement de la pensée rationnelle" [Hilbert 1926, 222-223]. Il s'agira de donner un fondement aux mathématiques classiques en faisant l'économie de l'hypothèse de l'infini actuel. Le but de Hilbert est d'utiliser des raisonnements finitistes pour donner un fondement aux raisonnements classiques. Hilbert accorde à Brouwer que les raisonnements classiques, comme ceux qui utilisent le tiers exclu et font intervenir un infini actuel, n'ont pas d'évidence propre. Pour prendre une évidence propre, un raisonnement ne doit faire intervenir qu'un nombre fini, quoique illimité, d'objets. De tels raisonnements constituent une mathématique finitiste. Ils n'ont pas à être justifiés, ils sont évidents et pourront être utilisés pour donner un fondement aux mathématiques classiques.

Plusieurs remarques s'imposeraient sur la délimitation de la mathématique finitiste. Techniquement, ses limites sont plus strictes que celles de la mathématique intuitionniste. Mais, surtout, sa justification n'est pas la même. Brouwer appuyait sa critique du raisonnement classique sur l'idée de constructions dans le temps. Les propositions mathématiques reposent sur les opérations d'une conscience et ces opérations doivent pouvoir être réalisées dans le temps. Ainsi, pour délimiter la mathéma- 
tique intuitionniste, Brouwer interroge ce que peut la conscience. Hilbert reproche à Brouwer de faire tomber les mathématiques dans le subjectif et l'arbitraire. La délimitation de la mathématique finitiste doit garder une "signification objective" [Hilbert 1927, 133], [Hilbert \& Bernays 1934, 33]. En fait, pour Hilbert, le raisonnement doit porter sur des objets qui peuvent être présentés dans l'expérience, ce qui implique qu'ils soient en nombre fini. Ce critère d'évidence est rapporté à Kant : "Nous sommes d'accord avec les philosophes, spécialement avec Kant. Celui-ci avait déjà pour doctrine que les mathématiques ont un contenu indépendant de la logique [...]. La condition préalable de l'application des inférences logiques et de l'effectuation d'opérations logiques est l'existence d'un donné dans la perception : à savoir l'existence de certains objets concrets extra-logiques qui en tant que sensations immédiates précèdent toute pensée. Pour que le raisonnement soit sûr, il faut que ces objets soient perçus dans toutes leurs parties et que leur occurrence, leur caractère distinct, leur succession, leur juxtaposition se présentent à l'intuition [...]" [Hilbert 1926, 228] Ces objets, dans l'arithmétique finitiste, sont des bâtons, des barres verticales tracées sur une feuille de papier. Les raisonnements dépendent de manipulations sur les séries de barres verticales : mettre bout à bout deux séries, en comparer la longueur, etc. Dans tous les cas, l'évidence d'un raisonnement tient à ce qu'il porte sur des objets concrets, qui se laissent présenter dans l'intuition. Finalement, Hilbert justifie l'évidence des raisonnements finitistes en renvoyant à "l'expérience concrète" et, de fait, à l'intuition de l'espace.

Cela dit, il s'agira d'utiliser les raisonnements finitistes pour donner un fondement aux mathématiques classiques, qui outrepassent les limites finitistes. Les mathématiques transfinies seront formalisées et représentées par des manipulations de signes dépourvus de sens. Partant d'une théorie donnée, on commence par faire l'inventaire des signes utilisés, analogue à un alphabet. On donne des règles, analogues à l'orthographe et à la grammaire, pour constituer à partir de ces signes des formules. On donne des axiomes qui serviront de prémisses dans les déductions. On donne des règles pour déduire une formule d'une autre. Ces règles ne s'appliquent qu'aux signes figurant dans les formules et ne prennent pas en considération le sens que pourraient posséder ces signes. Le raisonnement est remplacé par une manipulation de signes. La théorie est remplacée par "un stock de formules", construites et enchaînées selon des règles explicites [Hilbert 1926, 233]. Une démonstration se présente comme un dessin conforme à des règles convenues.

Ainsi, Hilbert distingue une mathématique finitiste, dont les raisonnements possèdent une évidence propre mais sont soumis à des res- 
trictions, et une mathématique formelle, qui consiste en manipulations symboliques selon des règles convenues. Il s'agira de démontrer la noncontradiction des théories formelles au moyen de raisonnements finitistes. Pour cela, on raisonnera sur les dessins, qui représentent les démonstrations dans un système formel. Une contradiction se manifesterait par un dessin ayant pour conclusion une ligne de la forme 0 ?0. On analysera les dessins conformes aux règles convenues pour démontrer que de tels dessins ne peuvent pas s'achever sur une ligne comme 0 ?0, en se limitant dans ces raisonnements à des inférences finitistes. Ces raisonnements sur les démonstrations mathématiques constituent une métamathématique. La métamathématique doit donner un fondement à la mathématique : établir, au moyen de raisonnements finitistes, qui, possédant une évidence propre, n'ont pas à être justifiés, que les raisonnements mathématiques s'effectuent sans contradiction. Pour fonder les raisonnements classiques, il n'est pas nécessaire d'affirmer la réalité des collections infinies sur lesquelles ils portent ou de prêter à la conscience mathématicienne la faculté de réaliser des processus transfinis. En fait, le programme formaliste fait de l'infini "quelque chose d'apparent", une sorte de phénomène bien fondé, que l'on peut utiliser en mathématiques sans lui reconnaître aucune réalité [Hilbert 1926, 221].

Hilbert semble renvoyer le fondement des mathématiques à l'intuition de l'espace. L'espace intervient deux fois dans le programme de fondement. Une première fois, l'espace représente la garantie des raisonnements finitistes. Ceux-ci prennent une évidence propre dans la mesure où leurs objets se laissent présenter dans l'expérience et dans l'espace. Ici, l'espace joue un rôle analogue à celui du temps dans l'intuitionnisme de Brouwer. Comme la possibilité d'une réalisation dans le temps pour l'intuitionnisme, la possibilité d'une présentation des objets dans l'espace est, pour le formalisme, la marque de l'évidence d'un raisonnement et la source de ses limitations. Une deuxième fois, l'espace est le milieu dans lequel doivent être représentées les démonstrations mathématiques pour faire l'objet de la méta-mathématique et être prouvées consistantes, c'est-à-dire être fondées. Bref, alors que Brouwer fondait les mathématiques sur des constructions dans le temps, en rejetant la possibilité de constructions dans l'espace, Hilbert semble revenir à ce qui serait une construction dans l'espace. Hilbert rattache le fondement des mathématiques à l'intuition de l'espace.

Néanmoins, Hilbert reprend l'idée du temps. En fait, Hilbert est confronté à la difficulté suivante. Brouwer objecte que ces stocks de formules, ces dessins conformes à des règles convenues, par lesquels Hilbert remplace les mathématiques transfinies, n'ont pas de sens et ne consti- 
tuent qu'un jeu gratuit, qu'il est inutile de chercher à fonder. Hilbert veut montrer que ce jeu de formules reflète la pensée mathématique, de sorte que la métamathématique qui prend pour objet ce jeu de formule réalise une analyse et, finalement, la fondation de la pensée mathématique. L'idée de fonder les mathématiques en prouvant la consistance d'un jeu de formules suppose que ce jeu de formules reflète les systèmes de pensées, qui constituent les mathématiques. Ainsi, Hilbert est conduit à postuler une sorte d'isomorphisme entre la pensée, dans l'esprit des mathématiciens, et son expression comme formule, sur le papier. Les formules sont les "images" de nos pensées [Hilbert 1923, 133]. Les règles qui gouvernent l'enchaînement des formules correspondent à celles qui gouvernent l'enchaînement de nos pensées. La formalisation, qui explicite ces règles, devient une enquête sur les règles, le fonctionnement de notre esprit :

"Procéder axiomatiquement, c'est simplement avoir conscience de sa pensée" [Hilbert 1922, 115]. "L'idée maîtresse de ma théorie de la démonstration n'est rien d'autre que de dépeindre l'activité de notre intelligence, de dresser un inventaire des règles d'après lesquelles notre pensée fonctionne réellement. La pensée est parallèle à la langue et à l'écriture, elle procède en formant et en alignant des phrases à la suite" [Hilbert 1927, $158]$.

"Ma théorie de la démonstration ne fait que simuler l'activité interne de notre entendement et enregistrer un procès-verbal des règles d'après lesquelles fonctionne notre pensée. En effet, la pensée procède parallèlement aux actes de parler et d'écrire : en formant et en alignant linéairement des phrases" [Hilbert 1930, 195].

Hilbert veut établir la portée de sa théorie de la démonstration : portée scientifique en ce qu'elle vise à établir le fondement des mathématiques; portée philosophique, puisqu'elle semble permettre d'interroger le fonctionnement de l'esprit. Mais cette double portée dépend du parallélisme, de l'isomorphisme postulé entre pensées et formules. Or celui-ci exige la linéarité du formalisme : à savoir, pour paraphraser Hilbert, que les démonstrations soient composées de formules disposées les unes à la suite des autres ou, autrement dit, que les règles d'inférence définissent une relation d'ordre totale entre les formules d'une démonstration. Cette linéarité semble renvoyer à la temporalité de la pensée. Si les formules reflètent les pensées et si les pensées s'enchaînent dans le temps, venant les unes à la suite des autres, il faut que les formules puissent, parallèlement, s'écrire les unes à la suite des autres. Je laisse de côté la question de savoir si le formalisme, qu'utilise Hilbert, est, en effet, linéaire. Ce que je veux souligner, c'est que Hilbert exige la linéarité de son formalisme 
et que la linéarité du formalisme traduit la temporalité de la pensée. Dans cet isomorphisme entre pensées et formules, une relation d'ordre totale entre les formules se traduit par une relation d'ordre totale entre les pensées. Finalement, l'idée que le temps est la forme de notre vie mentale, de notre pensée intérieure, resurgit dans le programme hilbertien et, là encore, elle est corrélative de restrictions pour les possibilités mathématiques : la linéarité du formalisme.

Revenons sur le rôle de l'espace et du temps dans l'intuitionnisme et dans le formalisme. D'emblée, Brouwer fonde les mathématiques sur l'intuition du temps : la possibilité d'une réalisation temporelle est le fondement des opérations et la source des limitations de la mathématique intuitionniste. Hilbert fonde les mathématiques sur l'intuition de l'espace : d'une part, la possibilité d'une présentation de leurs objets dans l'espace marque l'évidence des raisonnements finitistes ; d'autre part, la possibilité de représenter les démonstrations comme des figures dans l'espace permet d'en faire des objets pour la méta-mathématique. Néanmoins, Hilbert semble maintenir la thèse kantienne que le temps est la forme $a$ priori de la vie mentale, de la pensée intérieure. Cette thèse implique des restrictions pour les possibilités mathématiques : elle conduit à exiger la linéarité du formalisme. Ainsi, bien qu'ils mettent en place deux dispositifs conceptuels différents, Brouwer et Hilbert s'accordent pour inscrire le raisonnement mathématique dans le temps et déduire de là des conditions restrictives. Je voudrais maintenant évoquer plusieurs tentatives pour dégager le raisonnement mathématique de son inscription dans le temps et le rendre à l'espace. La première sera celle de Hilbert lui-même.

\section{3}

Bien avant son programme de fondement, Hilbert a défendu une conception du signe mathématique, qui s'oppose à ses thèses ultérieures, lesquelles ramènent la pensée à la temporalité et imposent la linéarité au formalisme. Je voudrais évoquer les quelques pages consacrées au rôle des signes, dans l'introduction de la conférence "Sur les problèmes futurs des mathématiques", donnée au Congrès international des mathématiciens de 1900. Les signes ne sont pas considérés en tant qu'expression de la pensée, mais dans leur rôle heuristique. En effet, le progrès mathématique passe par un travail, un jeu et, du moins, l'usage de ce que, d'emblée, Hilbert considère comme des symboles au même titre : chiffres lorsque l'on pose une opération arithmétique; signes algébriques comme les parenthèses sans lesquels on ne pourrait calculer des expressions gé- 
nérales; courbes, intervalles emboîtés en analyse; et, bien entendu, les figures du point, de la droite et du cercle en géométrie. Hilbert n'a pas besoin d'insister sur l'usage des figures en géométrie. Il s'agit de montrer que les symboles arithmétiques, algébriques ont le même rôle que les figures géométriques. Cela vient de ce que ces signes ont une sorte d'effet suggestif. Le mathématicien est sollicité par la formule qu'il écrit. Pour résoudre une équation, démontrer une proposition, il travaillera sur la formule, tentera de la transformer, dans un sens ou dans un autre, et c'est dans ce jeu sur les symboles que se dessine une voie. "Lorsque pour la première fois nous attaquons un problème en Arithmétique comme en Géométrie, nous tentons de rapides combinaisons, inconscientes et souvent mal assurées, nous confiant à un certain sentiment quant au comportement des signes arithmétiques, dont nous ne pourrions pas plus nous passer que de la faculté de voir dans l'espace en géométrie" [Hilbert 1900, 9]. Ainsi, les symboles arithmétiques, par exemple, ont la fécondité, la même efficace et, finalement, le même rôle que les figures géométriques. Ils guident le travail du mathématicien. Cependant, Hilbert donne aux figures géométriques et aux signes linéaires, aux formules arithmétiques, non seulement le même rôle mais également le même statut. Il y a une analogie parfaite entre les figures en géométrie et les formules en arithmétique, au point que l'on peut faire de la formule une figure et de la figure une sorte de formule : "Les signes et les symboles de l'Arithmétique sont des figures écrites et les figures géométriques sont des formules dessinées" [Hilbert 1900, 8]. La difficulté, pour tenir cette analogie, est que, apparemment, les symboles, en arithmétique, sont utilisés selon des règles, règles qui indiquent comment former et transformer une formule, comment poser une addition et calculer la somme, alors que l'usage des figures, en géométrie, ne semble reposer que sur notre intuition de l'espace. Or c'est ce point que Hilbert conteste. Les figures en géométrie dépendent de règles implicites : "De même que dans l'addition de deux nombres on ne doit pas poser les chiffres les uns sous les autres d'une façon inexacte, mais au contraire appliquer exactement les règles de calcul, c'est-à-dire les axiomes de l'Arithmétique, de même les opérations sur les symboles géométriques doivent être déterminées au moyen des axiomes de la Géométrie et de leur association" [Hilbert 1900, 9]. En fait, il s'agit d'expliciter les règles qui nous permettent de construire et de transformer les figures, de les fixer dans un système d'énoncés, des axiomes, pour faire de la construction géométrique une méthode de preuve, susceptible d'établir un théorème de façon aussi rigoureuse qu'une dérivation logique. 
En somme, Hilbert donne le même statut aux formules et aux figures : ce sont des systèmes de symboles gouvernés par des règles d'emploi et qui constituent des preuves rigoureuses lorsque leurs règles sont explicitées. Cette thèse mériterait d'être étayée plus longuement. Le rôle des règles, par rapport à l'intuition de l'espace, n'est pas clair en géométrie. ${ }^{1}$ Quoi qu'il en soit, les figures géométriques apparaissent comme un formalisme, un système de signes et de règles d'emploi, non linéaire. Ainsi, Hilbert se trouve envisager la possibilité d'un formalisme qui n'est plus soumis à l'exigence de linéarité et où le rapport entre "formules" et pensées reste en question. Hilbert esquisse une conception de la logique beaucoup plus large que celle qu'il défendra dans le cadre de son programme fondationnel. Il est vrai que ce texte, au Congrès de 1900, garde un caractère délibératoire et ne prétend pas donner des principes normatifs pour le développement logique comme les textes des années 1920, qui mettent en place le programme fondationnel. Ceux-ci font l'hypothèse d'un isomorphisme entre pensées et formules, et exigent la linéarité du formalisme, corrélative de la temporalité de la pensée. Finalement, la question est de savoir comment sortir de ce dispositif. Peut-on mettre en doute la temporalité de la pensée ou faut-il refuser de rapporter l'enchaînement des formules à un enchaînement de pensées? Nous verrons deux exemples, avec Frege et Gentzen.

Frege ne met pas en doute la temporalité de la pensée mais rejette l'idée que les écritures logiques doivent reproduire cette forme psychologique. Dans plusieurs textes, au début des années 1880, Frege défend son Idéographie contre une série des critiques. Or l'une des particularités, qui, du reste, fait la lourdeur de l'idéographie et qui, finalement, amènera à son abandon au profit de notations plus maniables, est que les formules logiques se trouvent représentées par des figures bi-dimensionnelles. En effet, la stratégie de Frege consiste à définir les connecteurs du calcul des propositions au moyen de l'implication et de la négation. Or une implication $A \rightarrow B$ est représentée comme l'illustre la figure 1 (voir page 212).

La négation est symbolisée par un trait vertical. Très vite, la figuration d'une formule complexe devient encombrante. Ainsi, $C \rightarrow(A \wedge B)$ se représente comme l'illustre la figure 2 (voir page 212).

Frege s'efforce de justifier l'usage de figures bi-dimensionnelles, contre le reproche que lui fait par exemple Schröder "d'avoir sacrifié à la coutume japonaise de l'écriture verticale" [Frege 1882-1883, 77]. Il s'agit de

1. Cavaillès, par exemple, développe l'analogie entre figures et formules en introduisant la notion d'espace combinatoire (cf. [Cavaillès 1938, 101], [Cassou-Noguès 2001-b, 102 et suivantes]). 


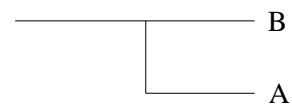

Figure 1 - Représentation de l'implication $A \rightarrow B$.

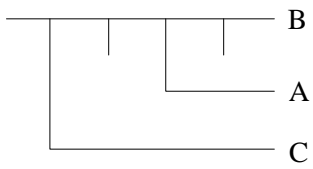

Figure 2 - Représentation de $C \rightarrow(A \wedge B)$. Les traits verticaux symbolisent la négation. 
comparer les mérites des signes parlés, ou des signes phonétiques, qui se suivent dans le temps et seraient linéaires, et des signes écrits, des figures qui peuvent utiliser les deux dimensions de la feuille de papier. Frege reconnaît "l'affinité très étroite des sons et des faits de conscience" [Frege 1882, 66]. Cette affinité vient d'abord de "la forme en laquelle ils se manifestent, la succession temporelle, [qui] leur est commune" [Frege $1882,66]$. Il faut reconnaître que les signes linéaires, qui se suivent dans le temps, sont isomorphes à nos pensées dans leur succession psychologique. Par ailleurs, la voix, avec ces modulations infinies, se prête à l'expression des subtiles variations de nos sentiments.

Pourtant, ces caractères, qui font l'affinité des signes parlés à la conscience psychologique, font leur défaut pour l'expression logique. En effet, parce qu'ils sont modulables, variables, les signes parlés n'auront pas la netteté des figures et leur univocité. D'autre part, et surtout, si les signes parlés reproduisent la chronologie linéaire des pensées, il n'en reste pas moins que l'addition d'une nouvelle dimension rend plus facile l'expression des rapports logiques. En réalité, l'infériorité des signes parlés vient de ce que, si nos pensées se succèdent dans le temps, il n'y a pas de raison à ce que leurs rapports logiques s'expriment dans le même ordre linéaire : "la simple disposition en une série linéaire ne correspond nullement à la multiplicité des rapports logiques suivant lesquels les pensées sont liées les unes aux autres" [Frege 1882, 67]. En réalité, l'existence d'une structure commune des signes parlés avec le fil de notre pensée devient un défaut. Elle nous empêche de dépasser ces rapports contingents entre nos pensées pour accéder à leurs rapports logiques. "Le fait que les signes sonores épousent si étroitement les conditions corporelles et psychiques de la raison a peut-être justement l'inconvénient de maintenir ces signes dans leur dépendance" [Frege 1882, 66-67].

Le cadre de ces remarques de Frege est analogue à celui dans lequel Hilbert place son programme fondationnel. Frege note "la linéarité" des signes parlés, ou des signes phonétiques, et leur isomorphisme avec la pensée, dans sa succession chronologique. Cependant, à la différence de Hilbert, Frege justifie l'usage de figures, bi-dimensionnelles, en distinguant les conditions psychologiques de l'exercice de la pensée, la forme temporelle linéaire, des rapports logiques entre les propositions qui s'exprimeront dans le plan.

Les figures de l'Idéographie sont peu maniables et seront abandonnées au profit des notations linéaires des Principia Mathematica. Il faut attendre les recherches de Gentzen sur la déduction naturelle pour voir réapparaître des figures en logique. Or, paradoxalement, le but qui guide Gentzen est de reproduire la pensée des mathématiciens, dans sa réalité 
psychologique. Gentzen est un élève de Hilbert et se place dans le cadre hilbertien.

$\mathrm{Au}$ départ, Gentzen commence par une critique adressée à Hilbert. Gentzen refuse que l'enchaînement des formules dans les systèmes formels qu'utilise Hilbert puisse refléter la pensée véritable des mathématiciens. En effet, dans ces systèmes, les démonstrations partent des axiomes, alors que, en pratique, les mathématiciens raisonnent à partir d'hypothèses quelconques, tantôt pour déduire des propositions qui restent conditionnelles, tantôt pour tester ces hypothèses et, finalement, démontrer des propositions vraies. Ainsi, les démonstrations formalisées dans les systèmes hilbertiens déforment la pensée véritable des mathématiciens. Et Gentzen se donne pour tâche "d'approcher" et de "reproduire" les "raisonnements réels", dans un système ou un "calcul" de "déduction naturelle".

On le sait, la déduction naturelle a une forme d'arbre. Les hypothèses, dont part la déduction, représentent la terminaison des branches au sommet de l'arbre. Tantôt les règles d'inférences (par exemple, l'introduction de "ou") permettent de déduire une nouvelle formule à partir d'une unique formule, ce qui, dans l'arbre de la déduction, fait descendre le long d'une branche. Tantôt les règles d'inférences (par exemple, l'introduction de "et") permettent de déduire une nouvelle formule à partir de deux formules déjà obtenues, ce qui correspond, dans l'arbre de la déduction, à un nIJud où se rejoignent plusieurs branches. Enfin, on peut éliminer des hypothèses utilisées dans la déduction de façon, finalement, à démontrer une formule vraie. Celle-ci est la base de l'arbre.

Chaque branche de l'arbre, tant que l'on se contente de déduire une formule d'une autre, est linéaire. Cependant, pour appliquer une inférence partant de plusieurs formules, il faut considérer que ces formules sont données ensemble et, par conséquent, que l'enchaînement procède simultanément sur les branches de l'arbre qui se rejoignent. Si chaque branche est linéaire, l'enchaînement s'effectuant simultanément sur plusieurs branches, l'arbre serait multilinéaire. Ici, les règles d'inférence ne définissent pas une relation d'ordre totale entre les formules d'une déduction naturelle. Bref, la déduction naturelle n'est pas linéaire et, si elle reflète un enchaînement de pensées, celui-ci n'est pas temporel au sens d'un ordre total entre les pensées. Cependant, Gentzen veut que les déductions naturelles reproduisent la pensée réelle du mathématicien. Or, dans le dispositif hilbertien, l'expression ne peut reproduire la pensée qu'à la condition d'être linéaire. Gentzen demande dans quelle mesure, puisqu'elles ne sont pas linéaires, les déductions naturelles peuvent refléter la pensée mathématique. Plus précisément : 
"En demandant que les formules soient arrangées en forme d'arbre, nous nous écartons quelque peu de l'analogie avec le raisonnement réel [qui] comporte nécessairement une séquence linéaire de propositions à cause de l'ordre linéaire de nos expressions verbales [... ]" [Gentzen 1935, 184]. ${ }^{2}$

Gentzen semble reprendre deux thèses de Hilbert : le parallélisme entre la pensée et son expression ; la temporalité, une relation d'ordre totale, dans l'enchaînement des pensées. Dans ce contexte, la non-linéarité de la déduction naturelle en fait une image déformante de l'enchaînement des pensées. Plus précisément, Gentzen commence par reconnaître un parallélisme entre l'expression et la pensée. Il applique un isomorphisme pour déduire de la linéarité de la parole la linéarité de la pensée : le raisonnement réel est linéaire "à cause de" la linéarité de l'expression verbale. D'autre part, Gentzen reconnaît la non-linéarité de la déduction naturelle mais refuse de lui appliquer l'isomorphisme. La remarque de Gentzen témoigne de ce que J. Derrida appelle le privilège de la voix [Derrida 1967]. En effet, c'est le privilège de la voix sur l'écrit, le mythe de la parole, qui conduit Gentzen à appliquer l'isomorphisme à l'expression linéaire et à refuser de l'appliquer à la déduction naturelle, bien que, au départ, celle-ci soit conçue pour traduire la pensée véritable. Finalement, pourquoi considérer que la voix donne une meilleure image de la pensée que les déductions naturelles? Pourquoi ne pas reconnaître aux déductions naturelles la fonction pour laquelle elles ont été constituées, refléter la pensée véritable des mathématiciens? Cela serait incompatible avec la linéarité supposée, c'est-à-dire la temporalité, de l'enchaînement des pensées. ${ }^{3}$

La position du temps comme forme de la pensée est commune à Brouwer et au Hilbert des années vingt. Dans le cadre du programme hilbertien, elle devrait imposer la "linéarité" du formalisme. Il devrait exister une relation d'ordre, totale et naturelle, entre les formules d'une démonstration, l'ordre des formules correspondant à la succession des pensées dans l'esprit mathématicien. Or Frege, qui se trouvait faire face à un problème analogue, puis Gentzen, avec ses déductions naturelles, imaginent des systèmes logiques dans lesquels cette relation d'ordre n'existe pas. Frege puis Gentzen reconnaissent l'existence d'un isomorphisme entre la pensée et l'expression verbale mais veulent placer leur système logique hors du domaine d'application de cet isomorphisme, de la pensée à l'expression. Ils détachent le raisonnement mathématique, dans la mesure où il s'exprime dans un tel système, de la forme du temps dans laquelle,

2. "Expressions verbales" traduit l'allemand "Aussagen" ou l'anglais "utterances".

3. Ce point est développé dans [Cassou-Noguès 2001a]. 
à la suite de Kant et de Brouwer, le maintient Hilbert. Ils ouvrent une nouvelle voie pour le fondement des mathématiques, qui ne réfère pas la preuve au temps mais à l'espace.

Or on retrouve, dans la logique contemporaine, l'opposition entre ces deux perspectives, l'une qui situe les preuves dans le temps et l'autre qui situe les preuves dans l'espace. Je m'appuie sur les analyses de G. Longo pour évoquer deux exemples [Longo 1995], [Longo 2003]. En premier lieu, dans la théorie des automates, on peut isoler les machines de Turing, comme des automates essentiellement temporels, et les opposer aux automates cellulaires, de Hebb, de von Neumann, aux réseaux connexionnistes ou aux systèmes concurrentiels. En effet, les machines de Turing ne dépendent pas de l'espace dans lequel elles sont situées. Il n'importe pas qu'elles soient représentées par un dispositif matériel situé dans un espace tridimensionnel, et pourvues d'un ruban, d'un espace de dimension 1, pour faire leurs calculs. Par exemple, elles ont la même puissance qu'elles disposent d'un ruban, d'un espace à une dimension, d'un plan ou d'un espace à $n$ dimensions. Seul importe, dans la définition des machines de Turing, le temps qui règle le calcul. En revanche, de l'autre côté, les réseaux cellulaires sont décrits par la répartition de leurs cellules dans l'espace et, souvent, justifiés par l'analogie avec la répartition des neurones dans le cerveau. En ce sens, les machines de Turing représenteraient des automates essentiellement temporels par opposition aux réseaux cellulaires fondés sur leur spatialité. D'autre part, les arguments épistémologiques visant à justifier certains systèmes logiques peuvent passer par une référence à l'espace et au temps. C'est le cas, par exemple, des logiques dialogiques, qui se présentent sous forme de dialogues et qui sont parfois justifiées par le caractère temporel de ces dialogues, qui les rapprocherait de la pensée naturelle. A l'inverse, l'un des arguments épistémologiques, de J.-Y. Girard et de G. Longo, pour défendre la logique linéaire puis la logique "ludique" et souligner leur nouveauté, est que les preuves y sont représentées par des réseaux dans l'espace. La logique ludique représenterait alors une tentative pour introduire l'idée de localisation, une spatialité ou une géométrie en logique.

Bref, la référence à l'espace et au temps conserve un rôle dans les arguments épistémologiques de la logique contemporaine. Elle reprend une réflexion, engagée par Kant, poursuivie par Frege, Borel, Brouwer, Hilbert et Gentzen, sur le lieu de la preuve mathématique et de son fondement. 


\section{Références}

BOREL, EMILE

1905 Quelques remarques sur les principes de la théorie des ensembles. Cité d'après [Rivenc \& Rouilhan 1992, 294-295].

Brouwer, Luitzen E. J.

1912 Intuitionism en formalism. Cité d'après la traduction française par Jean Largeault [Largeault 1992, 39-54].

Cassou-Noguès, Pierre

2001a Hilbert, Figures du savoir, Paris : Les Belles Lettres, 2001.

2001b De l'expérience mathématique. Essai sur la philosophie des sciences de Jean Cavaillès, Problèmes et controverses, Paris : Vrin, 2001.

Cavaillès, Jean

1938 Méthode axiomatique et formalisme. Essai sur le problème du fondement des mathématiques, Paris : Hermann, 1981.

DERRIDA, JACQUES

1967 La voix et le phénomène, Paris : P. U. F., 1967.

Frege, Gottlob

1879 Begriffsschrift, eine der arithmetischen nachgebildete Formelsprache des reinen Denkens, Halle : Nebert, 1879. Cité d'après la traduction française par M. A. Sinaceur in [Rivenc \& Rouilhan 1992, 98-129].

1882 Que la science justifie le recours à une idéographie. Cité d'après la traduction française par C. Imbert : Ecrits logiques et philosophiques, Paris : Seuil, 1971.

1882-1883 Sur le but de l'idéographie. Cité d'après la traduction française par C. Imbert : Ecrits logiques et philosophiques, Paris : Seuil, 1971.

Gentzen, Gerhard

1935 Untersuchungen über das logische Schließen, Mathematische Zeitschrift, 39 : 176-210 et 405-31.

Girard, JEAN-YVES

2001 Locus Solum, Mathematical Structures in Computer Science, $11(3)$. 
1900 Mathematische Probleme. Vortrag gehalten auf dem internationalen Mathematiker-Kongress zu Paris, 1900, Arch. der Math. und Physik, 1 : 44-63 et 213-237. Cité d'après la traduction française par L. Laugel : Sur les problèmes futurs des mathématiques, Sceaux : J. Gabay, 1990.

1922 Neubegründung der Mathematik, Erste Mitteilung, Abh. aus d. Math. Semin. d. Hamb. Univ., 1 : 157-177. Cité d'après la traduction française par Jean Largeault : Nouvelle fondation des mathématiques. Première communication, in [Largeault 1992, 107-131].

1923 Die logischen Grundlagen der Mathematik, Mathematische Annalen, 88 : 151-165. Cité d'après la traduction française par Jean Largeault : Les fondements logiques des mathématiques, in [Largeault 1992, 131-145].

1926 Ueber das Unendliche, Mathematische Annalen, 95. Cité d'après la traduction française par Jean Largeault : Sur l'infini, in [Largeault 1972, 220-245].

1927 Die Grundlagen der Mathematik, Abh. aus d. Math. Semin. d. Hamb. Univ., $6: 65-83$. Cité d'après la traduction française par Jean Largeault : Les fondements des mathématiques, in [Largeault 1992, 145-164].

1928 Probleme der Grundlegung der Mathematik, Mathematische Annalen, 102 : 1-9. Cité d'après la traduction française par Jean Largeault : Problème de fondation des mathématiques, in [Largeault 1992, 175-187].

1930 Die Grundlegung der elementaren Zahlenlehre, Mathematische Annalen, 104 :485-94. Cité d'après la traduction française par Jean Largeault : Le fondement de l'arithmétique élémentaire, in [Largeault 1992,187-197].

Hilbert, David \& Bernays, Paul

1934 Grundlagen der Mathematik I, Berlin : Springer, 1934.

Kant, Immanuel

1781-1787 Kritik der reinen Vernunft. Cité d'après la traduction française par A. Tremesaygues et B. Pacaud : Critique de la raison pure, Quadrige, Paris : P.U.F., 1990. 
LARGEAUlt, JEAN

1972 Logique mathématique, textes, Paris : A. Colin,1972.

1992 Intuitionisme et théorie de la démonstration, Paris : Vrin, 1992.

Longo, Giuseppe

1995 The difference between Clocks and Turing Machines, La Nuova Critica, 29 (1) : 31-42.

2003 Space and Time in the Foundations of Mathematics, Intellectica, 36-37 (1-2).

Rivenc, François \& Rouilhan, Philippe de 1992 Logique et fondement des mathématiques, Paris : Payot, 1992. 